\title{
791 MULTI-SPECIFIC GUIDANCE AND NAVIGATION CONTROL (GNC) T CELL ENGAGERS CONVERT PDL1 ADAPTIVE RESISTANCE TO DRUG SENSITIVITY
}

Jahan Khalili*, Mark Gilchrist, Sarah Rickli, Daphne Toglia, Yi Zhu. SystImmune Inc, Redmond, WA, United States

Background Major challenges to $\mathrm{T}$ cell engager drugs are antigen loss and adaptive resistance, often in the form of PDL1 upregulation on cancer cells or the tumor microenvironment. Engaged $\mathrm{T}$ cells are dependent on cancer cell co-stimulation, and synaptic engagements that may deteriorate the function of the effector $\mathrm{T}$ cell compartment.

Methods Guidance and Navigation Control (GNC) drugs are multi-specific cell engagers. This platform of GNC $\mathrm{T}$ cell engagers is designed to mediate direct cytolysis of cancer cells by $\mathrm{T}$ cells, and influence the outcome of synaptic engagement, overcome PDL1 resistance, and respond to biological signals in the tumor microenvironment to increase functional potency. GNC that bind tumor antigens, CD3, 4-1BB and PDL1 are designed to redirect $\mathrm{T}$ cells toward tumor antigens and engage PDL1 as a target antigen.

Results $\mathrm{T}$ cell activation resulting from tumor associated antigen and $\mathrm{CD} 3 / 41 \mathrm{BB}$ binding, results in IFN-gamma release. The local IFN-gamma upregulates PDL1 on cancer cells, increasing the antigen density and converting a major mechanism of adaptive resistance into local drug sensitivity. This novel design and first-in-class mechanism of action may reduce resistance mediated by both antigen loss, and immune suppression through cytolysis of PDL1 expressing cells within the tumor microenvironment.

Conclusions Knowledge of PDL1 regulation and its role as a universal target in the solid tumor microenvironment provides one path forward for multi-modal $\mathrm{T}$ cell engagers with potential for broad clinical efficacy. Multi-specific GNC demonstrated preclinical functionality, and developability. GNC T cell engagers targeting tumor associated and tumor specific antigens are currently in Phase I trials for solid and liquid cancer indications. ${ }^{1} 2$

\section{REFERENCES}

1. A Study of GNC-038, a Tetra-specific Antibody, in Participants With R/R NonHodgkin Lymphoma. ClinicalTrials.gov Identifier: NCT04606433.

2. A Study of GNC-039, a Tetra-specific Antibody, in Participants With Relapsed/ Refractory or Metastatic Solid Tumors. ClinicalTrials.gov Identifier: NCT04794972.

http://dx.doi.org/10.1136/jitc-2021-SITC2021.791 